\title{
Beyond Fibonacci patterns and the golden angle: phyllotactic variations and their cellular origin
}

\author{
Xiaofeng Yin ${ }^{1,2}\left(\right.$ D) Miho S. Kitazawa ${ }^{3,4}(\mathbb{0}$ \\ Published online: 28 April 2021 \\ (C) The Botanical Society of Japan 2021
}

Phyllotaxis, the regular arrangement of plant organs, has attracted researchers from different disciplines over centuries for its beauty (Adler et al. 1997; Barabé and Lacroix 2020; Jean and Barabé 1998). Such striking arrangement can be found at different levels in plants, including leaves, floral organs, flowers, and even inflorescences. Several types of phyllotactic patterns, exemplified by the Fibonacci patterns, are observed in all major land plant lineages, from non-vascular plants such as mosses, to seedless vascular plants such as lycophytes, and to seed plants such as angiosperms (Gola and Banasiak 2016).

Recent studies of phyllotaxis mainly focus on the mechanisms of the widespread Fibonacci patterns (Bhatia and Heisler 2018; Galvan-Ampudia et al. 2016; Kuhlemeier 2017; Sassi and Vernoux 2013; Shi and Vernoux 2019; Traas 2013). However, irregularities, stochasticities, and transitions of phyllotactic patterns are also frequently observed. For example, in the model plant Arabidopsis [Arabidopsis thaliana (L.) Heynh.], phyllotactic variations, temporal deviations, and transitions are all commonly observed during its ontogeny. Moreover, a recent study suggests that the

Xiaofeng Yin

yinx@g.ecc.u-tokyo.ac.jp

$\square$ Miho S. Kitazawa

kitazawa@celas.osaka-u.ac.jp

1 Department of Biological Sciences, Graduate School of Science, The University of Tokyo, Hongo 7-3-1, Bunkyo-ku, Tokyo 113-0033, Japan

2 Japan Society for the Promotion of Science, Tokyo, Japan

3 Center for Education in Liberal Arts and Sciences, Osaka University, 1-16 Machikaneyama-cho, Toyonaka, Osaka 560-0043, Japan

4 Department of Biological Sciences, Graduate School of Science, Osaka University, 1-1 Machikaneyama-cho, Toyonaka, Osaka 560-0043, Japan formation of Fibonacci patterns does not necessarily depend on the commonly assumed Golden Angle (Zhang et al. 2021a). In addition, only in recent years have we started to consider how phyllotaxis originated and evolved in land plants (Véron et al. 2021). All these aspects await to be investigated in more depths.

In this special issue of the JPR Symposium titled "Beyond Fibonacci Patterns and the Golden Angle: Phyllotactic Variations and their Cellular Origin", we aim to advance our understanding of phyllotaxis in these overlooked aspects. In particular we highlight patterns "outside" Fibonacci patterns and the evolutionary origins of phyllotaxis. Three review articles discussing general aspects (Yin 2021), stochasticities (Kitazawa 2021), and symmetries (Yonekura and Sugiyama 2021); and three original research articles focusing on pattern regeneration (Zhang et al. 2021b), a new system for studying phyllotactic transitions (Zagórska-Marek et al. 2021), and the origin of phyllotaxis (Kamamoto et al. 2021), are included in this special issue.

Phyllotaxis is an old research field in plant morphology yet still being explored and advanced continuously. Yin (2021) gave an overview on this fascinating phenomenon, covering a broad spectrum from the knowledge in classical literature to recent findings in the regulation of phyllotaxis using molecular genetics approaches. In the first part, phyllotactic patterns, parameters, transitions, and diversity were reviewed. In the second part, the molecular regulation mechanims of where and when a new plant organ emerges were discussed, focusing on the role of a plant phytohormone auxin; genetic factors participating in the regulation of the size, geometry, organization, and integrity of the apical meristem and thus contributing to phyllotactic variation and diversity were summarized; and the contributions of signals from developing organs, cell wall mechanics, and post apical meristem growth were also discussed. It was demonstrated that the morphological beauty of phyllotaxis is the 
result of many regulators exerting their respective effects in a coordinated fashion either directly or indirectly in the apical meristem.

Stochasticity exists ubiquitously and inevitably in biological processes, ranging from molecular processes such as gene expression, to cellular properties and dynamics, and to macroscopic phenotypes. Phyllotaxis is not an exception to such stochasticity. Kitazawa (2021) reviewed the developing fields connecting developmental stochasticity, phenotypic variation, and evolutionary diversification, with an aim to understand the diversity of floral phyllotaxis. Three categories of variation in vegetative and inflorescence phyllotaxis were suggested: fluctuations around the mean divergence angle, transitions between regular patterns, and temporal deviations from regular patterns called permutations. Some of the variations in floral phyllotaxis were similarly classified, and specialized features, such as deterministic development and coexistence of multiple types of organ, were discussed to show the flower-specific sources of variation. Since variation can be a driving force of evolution, studies on developmental stochasticity and variation will benefit the understanding of phyllotactic diversity.

Symmetry is an important component of geometric beauty and regularity. Phyllotactic patterns can be categorized by their symmetry, such as reflection symmetry, rotation symmetry, translation symmetry, corkscrew symmetry, and glide reflection symmetry. Yonekura and Sugiyama (2021) reviewed the classification of these phyllotactic symmetries according to the group theory, which was originally suggested by Yamada et al. (2004), and classified naturally occurring phyllotactic patterns according to their symmetry. The application of the group theory to phyllotactic symmetry by Yamada et al. (2004) also suggested a transition rule of phyllotactic patterns. Yonekura and Sugiyama (2021) further compared this rule with the developmental model of phyllotaxis (Yonekura et al. 2019), based on the mapping of simulation results in the parameter space of the model. Some of the transitions found in the developmental model were in accord with the transition rule suggested by Yamada et al. (2004) while some others were not, indicating that the transition rule was only partially supported by the developmental model. By classifying naturally occurring patterns by mathematical expression and examining mathematically proposed theories by models with different assumptions, this review showed an interplay between different disciplines.

One classical question in phyllotaxis research is how phyllotactic patterns are regenerated after an apical meristem is physically disturbed. By applying either mechanical wounding or finely controlled laser ablation, Zhang et al. (2021b) elegantly revealed the process of phyllotactic pattern regeneration in the inflorescence meristems of Gerbera hybrida cv. Terra Regina. Upon disrupted expression of $G h C L V 3$, a marker gene indicating the undifferentiated state of the inflorescence meristem, following physical disturbances, a rim comprising three to five layers of elongated cells was formed. Strikingly, this wounding rim had a low auxin signaling output. This was in sharp contrast to the neighboring region away from the wounding rim which had a high auxin signaling output. New auxin maxima formed regularly in this region and joined the existing ones to form parastichies, thus completing the pattern regeneration process. This work lays the foundation of future studies to understand the molecular mechanisms of de novo pattern formation upon physical disturbance.

The most popular model plant, Arabidopsis, does not have sufficient phyllotactic variations. Plants known for their great phyllotactic variations, such as Magnolia, Torreya, and Abies, are not suitable for experimental work. Therefore, to study the diversity of phyllotaxis beyond Fibonacci patterns, a new model system is required. Zagórska-Marek et al. (2021) showed that common verbena (Verbena officinalis L.) has preferable features in this regard. This perennial plant is suitable for laboratory experiments, and has phyllotactic transitions not only between growth phases but also within the indeterminate inflorescence axis, without changing the identity of lateral organs. Using in silico experiments, they suggested that phyllotactic transitions in the inflorescence might be triggered by the cumulative effect of fluctuations in the continuously decreasing bract primordia size. More importantly, they found that phyllotactic changes in the primary vascular system preceded those in the apical meristem. Their findings suggest the complexity of various systems to coordinate phyllotaxis and common verbena as a potential useful system to study phyllotactic transitions.

The origin of phyllotaxis in land plants remains a mystery. Using three moss species and combining histological and mathematical approaches, Kamamoto et al. (2021) carefully addressed this question. Unlike the well understood angiosperms, moss gametophore leaves and thus phyllotaxis are generated by creating new cutting faces in the tetrahedral apical initial cell. In the tested moss species, divergence angle in older merophytes converged to a species-specific value, which reflects distinct phyllotactic patterns at maturity. More importantly, a mathematical model demonstrated that the converged divergence angle was identical to the apical initial cell's division plane rotation angle regardless of the phyllotactic pattern. The model further predicted the inevitable and transient deviations of divergence angle in younger merophytes due to a shift in the centroid of the apical initial cell after a cell division. Together, it was shown that moss phyllotaxis is regulated by the division plane rotation angle and the shape of the apical initial cell, revealing the origin of phyllotaxis in and plants.

Despite the long history of phyllotaxis studies, there are still many unsolved questions. New discoveries are continuously being made through examining various new plant 
systems, revisiting classical knowledge, and incorporating new technologies and tools. We believe that investigating beyond Fibonacci patterns and the Golden Angle would enable us to have a thorough understanding of phyllotaxis.

Acknowledgement This special symposium is part of a fruitful discussion during the 83th Annual Meeting of Botanical Society of Japan. We would like to thank all authors and reviewers for their efforts. We would also like to thank Dr. Masako Mishio for the editorial support.

\section{References}

Adler I, Barabé D, Jean RV (1997) A history of the study of phyllotaxis. Ann Bot 80:231-244

Barabé D, Lacroix C (2020) Phyllotactic patterns: a multidisciplinary approach. World Scientific, Singapore

Bhatia N, Heisler MG (2018) Self-organizing periodicity in development: organ positioning in plants. Development 145: dev149336

Galvan-Ampudia CS, Chaumeret AM, Godin C, Vernoux T (2016) Phyllotaxis: from patterns of organogenesis at the meristem to shoot architecture. Wiley Interdiscip Rev Dev Biol 5:460-473

Gola EM, Banasiak A (2016) Diversity of phyllotaxis in land plants in reference to the shoot apical meristem structure. Acta Soc Bot Pol 85:3529

Jean RV, Barabé D (1998) Symmetry in plants. World Scientific, Singapore

Kamamoto N, Tano T, Fujimoto K, Shimamura M (2021) Rotation angle of stem cell division place controls spiral phyllotaxis in mosses. J Plant Res. https://doi.org/10.1007/s10265-021-01298-0

Kitazawa MS (2021) Developmental stochasticity and variation in floral phyllotaxis. J Plant Res. https://doi.org/10.1007/ s10265-021-01283-7
Kuhlemeier C (2017) Phyllotaxis. Curr Biol 27:R882-R887

Sassi M, Vernoux T (2013) Auxin and self-organization at the shoot apical meristem. J Exp Bot 64:2579-2592

Shi B, Vernoux T (2019) Patterning at the shoot apical meristem and phyllotaxis. Curr Top Dev Biol 131:81-107

Traas J (2013) Phyllotaxis. Development 140:249-253

Véron W, Vernoux T, Coudert Y (2021) Phyllotaxis from a single apical cell. Trends Plant Sci 26:2

Yamada H, Tanaka R, Nakagaki T (2004) Sequences of symmetrybreaking in phyllotactic transitions. Bull Math Biol 66:779-789

Yin X (2021) Phyllotaxis: from classical knowledge to molecular genetics. J Plant Res. https://doi.org/10.1007/s10265-020-01247-3

Yonekura T, Sugiyama M (2021) Symmetry and its transition in phyllotaxis. J Plant Res. https://doi.org/10.1007/s10265-021-01308-1

Yonekura T, Iwamoto A, Fujita H, Sugiyama M (2019) Mathematical model studies of the comprehensive generation of major and minor phyllotactic patterns in plants with a predominant focus on orixate phyllotaxis. PLoS Comput Biol 15:e1007044

Zagórska-Marek B, Turzańska M, Chimiel K (2021) Verbena officinalis Verbenaceae (Lamiales): a new plant model system for phyllotaxis research. J Plant Res. https://doi.org/10.1007/s10265-021-01288-2

Zhang T, Cieslak M, Owens A, Wang F, Broholm SK, Teeri TH, Elomaa P, Prusinkiewicz P (2021a) Phyllotactic patterning of gerbera flower heads. Proc Natl Acad Sci U S A 118:e2016304118

Zhang T, Wang F, Elomaa P (2021b) Repatterning of the inflorescence meristem in Gerbera hybrida after wounding. J Plant Res. https:// doi.org/10.1007/s10265-021-01253-z

Publisher's Note Springer Nature remains neutral with regard to jurisdictional claims in published maps and institutional affiliations. 